\title{
METASTABLE STATES IN STRONGLY COUPLED QUANTUM SYSTEMS WITH CONTINUOUS SPECTRA
}

\author{
LEWIS H. NOSANOW ${ }^{*}$ ) \\ Instituut voor theoretische fysica der Rijksuniversiteit, Utrecht, Nederland
}

\begin{abstract}
Synopsis
The problem of constructing metastable states in strongly coupled quantum systems whose spectra are continuous is treated within the context of the general perturbation method developed by Van Hove. The state is constructed in terms of the unperturbed states and its metastable character is proved without explicitly introducing the perturbed stationary states. An interesting feature of the result is that the construction of the state requires the use of a complex number, the real part of which is equal to the energy of the state while its imaginary part is juch larger than the line width of the state. The general result is applied to the Fermi gas and the Lee model. In the latter case it reduces to the result previously obtained by Glaser and Källén.
\end{abstract}

1. Introduction. Many systems in quantum physics are conveniently described in terms of metastable states, i.e. in terms of approximately stationary states which persist for a relatively long time before decaying into more complicated modes of motion. The classic example of such a system is that of an atom in interaction with the electromagnetic field, in which case the stationary states of the atom become metastable due to the effect of the field. The atom has a discrete spectrum in absence of the interaction and the methods for treating such a system were developed in the early days of quantum mechanics. In recent years, considerable attention has been devoted to systems whose spectra are continuous, e.g. interacting fields and many-body systems. The question of treating metastable states in such systems is a very natural one. Indeed the state of an unstable elementary particle or the state of a low-energy nucleon moving inside a large nucleus must both be regarded as metastable. The purpose of this paper is to treat a general system with a continuous spectrum within the context of perturbation theory and show under which conditions and by which method one can construct the state vector of a metastable state to general order in the interaction.

$\Lambda$ s the basis of our work, we shall usc the very general perturbation method for treating systems with continuous spectra which has been developed by Van Hove ${ }^{1}$ ). His method is especially well suited to the

*) Present address: Physics Department, University of California, La Jolla, California, U.S.A. 
investigation of the perturbed states of a system $\left.{ }^{2}\right)^{3}$ ). In fact, it yields a criterion which enables one to determine when it is possible to construct perturbed stationary states. When this criterion is not satisfied, i.e. when it is not possible to construct a perturbed stationary state corresponding to a given unperturbed state, it may be possible to construct a perturbed state which is metastable. The conditions under which this is possible have been given by $\mathrm{Hugenholtz}{ }^{4}$ ). He also gave an explicit construction for a metastable state; however, his construction must be considered as unsatisfactory because it leads to a state which can not be normalized.

To our knowledge, the only satisfactory treatments of metastable states not limited to lowest order perturbation theory have been given for the simple case of the Lee model. In particular, we wish to mention the work of Glaser and Källén ${ }^{5}$ ) who treated the Lee model for arbitrary coupling, because our general result reduces to theirs when applied to the Lee model. The method which they use is the most straightforward one possible. They make an ansatz for their metastable state and then expand it in terms of the true stationary states of the system, which are known for the Lee model. In this way they are able to select the best state vector for their state, and to calculate its energy, lifetime, and decay spectrum. Unfortunately this method can not be used in the general case because the complete set of perturbed stationary states is not known (in fact it is usually impossible to determine it even by perturbation methods). Thus the main difficulty which we had to overcome was to select our state vector and calculate its energy and lifetime without using the perturbed stationary states explicitly. It turns out that the extremely condensed mathematical form of Van Hove's approach makes this possible. Of course, it is not possible to calculate the decay spectrum without knowing the true stationary states. However, in the case that they are known, our method yields the decay spectrum in a simple way. An interesting feature of the general result is that the construction of the metastable state requires a complex number, the real part of which is the energy of the state while its imaginary part is much larger than the line width of the state.

We shall only mention very briefly other relevant work on this subject. Zumino ${ }^{6}$ ) and Höhler ${ }^{7}$ ) have treated the Lee model in the weak coupling case by using the analytical continuation method. We shall also use this method. Extensive work has been done by various Japanese authors $\left.\left.{ }^{8}\right)^{9}\right)^{10}{ }^{11}$ ), some of whom have only treated the Lee model and some of whom have treated the general case.

2. Formulation of the problem. We shall consider a system whose hamiltonian may be written as a sum of two terms

$$
H=H_{0}+H_{1} \text {. }
$$


The eigenstates and eigenvalues of $H_{0}$ will be assumed known, and $H_{1}$ will be treated formally by perturbation theory even though it is not. necessarily small. The perturbation treatment will be valid to general order. We shall use units such that $\hbar=1$. Our program is to make an ansatz for a state $\left|\psi_{\alpha}\right\rangle$ and then use perturbation theory to compute the quantity

$$
P_{\alpha}(t) \equiv\left\langle\psi_{\alpha}|\exp (-i t H)| \psi_{\alpha}\right\rangle
$$

which is the probability amplitude for finding the system in the state $\left|\psi_{\alpha}\right\rangle$ at the time $t$, if it was in this state initially. Suppose that after an approximate calculation we find that

$$
P_{\alpha}(t) \cong \exp \left(-i t E_{\alpha}\right)\left\langle\psi_{\alpha} \mid \psi_{\alpha}\right\rangle
$$

for times of the order of $\left(\operatorname{Im} E_{\alpha}\right)^{-1}$, where $E_{\alpha}$ is a complex energy such that

and

$$
\left|\operatorname{Re} E_{\alpha}\right| \gg\left|\operatorname{Im} E_{\alpha}\right| \text {, }
$$

$$
\operatorname{Im} E_{\alpha}-\left\{\begin{array}{l}
-\left|\operatorname{Im} E_{\alpha}\right| \text { for } t>0 . \\
+\left|\operatorname{Im} E_{\alpha}\right| \text { for } t<0 .
\end{array}\right.
$$

Then we may say that $\left|\psi_{\alpha}\right\rangle$ is a metastable state, and we may identify $\operatorname{Re} E_{\alpha}$ as the energy of the state and $\left(2 \operatorname{Im} E_{\alpha}\right)^{-1}$ as its lifetime. Needless to say, (2.3) can only result when the system satisfies certain conditions. These will be given in section 4 .

In order to establish an approximate result like (2.3), it is necessary to isolate a small parameter $\lambda$ in the problem. In fact, it usually turns out that $\operatorname{Re} E_{\alpha}$ and $\operatorname{Im} E_{\alpha}$ can be expressed in terms of a power series in $\lambda$. In our case it will be convenient to use $\lambda$ to reformulate all approximate expressions in terms of limiting processes, e.g. (2.4) will be reformulated as

$$
\lim _{\lambda \rightarrow 0} E_{\alpha}=E_{\alpha 0}
$$

where $E_{\alpha 0}$ is a real number. Let LIM be a short notation for a limiting process in which $\lambda \rightarrow 0,|t| \rightarrow \infty$, while the product $t\left(\operatorname{Im} E_{\alpha}\right)$ remains fixed. Since $\operatorname{In} E_{\alpha}$ is a power series in $\lambda$, holding the product $t\left(\operatorname{Im} E_{\alpha}\right)$ fixed means picking out the largest term in $\operatorname{Im} E_{\alpha}$ for small $\lambda$. We can now rewrite (2.3) as

$$
\operatorname{LIM}\left[\exp \left(i t \operatorname{Re} E_{\alpha}\right) P_{\alpha}(t)\right]=\exp \left(-|t|\left|\operatorname{Im} E_{\alpha}\right|\right)\left\langle\psi_{\alpha} \mid \psi_{\alpha}\right\rangle
$$

and this is actually what we shall prove. The absolute values of $t$ and $\operatorname{Im} E_{\alpha}$ enter into (2.7) because of (2.5). This formulation immediately implies that the state,

$$
\left|\psi_{\alpha}\right\rangle_{0}=\lim _{\lambda \rightarrow 0}\left|\psi_{\alpha}\right\rangle,
$$

must exist and must be a true stationary state of energy $E_{\alpha 0}$ for our system in the case $\lambda=0$. We shall adopt the convention that labeling a quantity with an extra subindex 0 refers to the value of the quantity when $\lambda=0$. 
If the system of interest is weakly coupled, we can identify $\lambda$ as the coupling constant. In this case, $\left|\psi_{\alpha}\right\rangle_{0}$ is simply an unperturbed eigenstate and every unperturbed eigenstate that does not go over into a scattering state will become metastable. However, for strongly coupled systems such as we wish to treat in this paper, $\lambda$ must have some other physical meaning, and $\left|\psi_{\alpha}\right\rangle_{0}$ can only be a perturbed stationary state. When we treat the case of a nucleon interacting with a large nucleus in section 8 , we shall find this parameter explicitly and explain its physical meaning.

3. Van Hove's method. In order to define $\left|\psi_{\alpha}\right\rangle$ and calculate $P_{\alpha}(t)$, we shall use Van Hove's method. His approach makes extensive use of the resolvent operator $R(z)$, which is defined by

$$
R(z)=\left(H_{0}+H_{1}-z\right)^{-1},
$$

where $z$ is an arbitrary complex number with the dimensions of energy. Using the well-known relationship between $\exp (-i t H)$ and $R(z)$, we may write

$$
P_{\alpha}(t)=\frac{i}{2 \pi} \oint \mathrm{d} z \exp (-i t z) \varphi_{\alpha}(z)
$$

where

$$
\varphi_{\alpha}(z)=\left\langle\psi_{\alpha}|R(z)| \psi_{\alpha}\right\rangle
$$

and the path of integration in (3.2) is a contour described in a counterclockwise direction around a sufficiently large portion of the real axis. Although it does not assume that $H_{1}$ is small, this treatment starts out by formally treating $H_{1}$ as a perturbation, whence (3.1) leads immediately to the series expansion

$$
R(z)=\sum_{n=0}^{\infty}(-1)^{n} \frac{1}{H_{0}-z}\left[H_{1} \frac{1}{H_{0}-z}\right]^{n},
$$

the simplicity of which is one of the most attractive features of the resolvent technique. Perhaps the most important assumption of this approach is that (3.4) is convergent for $z$ away from the real axis. The conditions under which this is valid are not known at present.

Now we shall introduce explicitly the representation in which $H_{0}$ is diagonal. We suppose that it has a continuous energy spectrum $\varepsilon_{\alpha}$ and eigenstates $|\alpha\rangle$ both labeled by a set of quantum numbers $\alpha$, some of which are continuous. We shall adopt the normalization.

$$
\langle\beta \mid \alpha\rangle=\delta(\beta-\alpha),
$$

where $\delta(\beta-\alpha)$ is a product of Dirac delta functions for the continuous quantum numbers and Kronecker symbols for the discrete ones. This notation is adapted to the continuous parameters, but could easily be 
completed to take account of the discrete ones. In order to calculate $P_{\alpha}(t)$, we shall have to know $\langle\beta|R(z)| \alpha\rangle$. This is calculated by making use of the property that matrix elements such as

$$
\left\langle\beta\left|\frac{1}{H_{0}-z}\left[H_{1} \frac{1}{H_{0}-z}\right]^{n}\right| \alpha\right\rangle
$$

can have a term proportional to $\delta(\beta-\alpha)$ even if the matrix element $\left\langle\beta\left|H_{1}\right| \alpha\right\rangle$ has no such singularity itself. We wish to emphasize that this diagonal singularity property depends strongly on the choice of the unperturbed part of the hamiltonian. Although this property has been general knowledge for a long time, it was $V$ an Hove who first showed that it had to be taken into account to all orders of the perturbation for systems with continuous spectra even for weak coupling.

When used in conjunction with (3.4), the diagonal singularity property allows one to calculate $\langle\beta|R(z)| \alpha\rangle$ by making an infinite number of partial summations. The result obtained by Van Hove may be written

$$
\langle\beta|R(z)| \alpha\rangle=\left[\langle\beta \mid \alpha\rangle+F_{\beta \alpha}(z)\right] D_{\alpha}(z),
$$

where $F_{\beta \alpha}(z)$ vanishes for $\beta=\alpha$ and

$$
D_{\alpha}(z)=\left[\varepsilon_{\alpha}-z-G_{\alpha}(z)\right]^{-1} .
$$

For the precise definitions of $F_{\beta \alpha}(z)$ and $G_{\alpha}(z)$, the reader is referred to the original papers $*$ ). As far as this paper is concerned, we are mainly interested in the fact that $\langle\beta|R(z)| \alpha\rangle$ is proportional to $D_{\alpha}(z)$, and that $D_{\alpha}(z)$ has the form given by (3.8). We shall also need to know the important analytic properties of these functions. Since $H$ is a hermitean operator, $\langle\beta|R(z)| \alpha\rangle$ can only have singularities on the real axis. It follows that $F_{\beta \alpha}(z), G_{\alpha}(z)$, and $D_{\alpha}(z)$ also have this property. If we let ${ }^{*}$ stand for the hermitean conjugate of an operator and the complex conjugate of a number, it also follows that

$$
\left[D_{\alpha}(z)\right]^{*}=D_{\alpha}\left(z^{\star}\right)
$$

and the same holds true for $G_{\alpha}(z)$. Again assuming the convergence of (3.4), it is possible to show that the only singularities of $F_{\beta \alpha}(z)$ and $G_{\alpha}(z)$ are finite discontinuities across the real axis from a finite point up to $+\infty$, i.e.

$$
\lim _{\eta \rightarrow 0} G_{\alpha}(x+i|\eta|) \neq \lim _{\eta \rightarrow 0} G_{\alpha}(x-i|\eta|)
$$

when $x$ is a point on the real axis where the function is singular.

The singularities of $D_{\alpha}(z)$ are a little more complicated. Clearly it has a finite discontinuity on the same portion of the real axis as $G_{\alpha}(z)$, which

\footnotetext{
*) See reference 1), section 3 or reference 4), section 4. The analytic properties of these functions are also derived here. A comprehensive summary of his own work has been given by $V$ an Hove in reference 12 , sections 2 and 4 .
} 
we call the cut of $D_{\alpha}(z)$. In addition this function can become infinite whenever the equation

$$
\varepsilon_{\alpha}-z-G_{\alpha}(z)=0
$$

has a solution on the real axis. It can be shown that (3.9) can have no solution for non-real $z$. We shall suppose that a real root of (3.9) exists when $\lambda=0$, and we shall call it $E_{\alpha 0}$. Further we shall suppose that the cut of $D_{\alpha}(z)$ begins at a point $a_{0}>0$, where $a_{0}$ is independent of $\lambda$. If $E_{\alpha 0}<a_{0}$, it is clear that $D_{\alpha 0}(z)$ has a pole at $E_{\alpha 0}$. However, if $E_{\alpha 0} \geq a_{0}$, then $D_{\alpha 0}(z)$ can not have a pole in the strict sense of the word because the discontinuity of $G_{\alpha 0}(z)$, although zero at $z=E_{\alpha 0}$, does not vanish in the neighbourhood of $E_{\alpha 0}$. Nevertheless, we shall still call $E_{\alpha 0}$ a pole of $D_{\alpha 0}(z)$, if it is possible to write

$$
D_{\alpha 0}(z)=\frac{N_{\alpha 0}}{E_{\alpha 0}-z}+\tilde{D}_{\alpha 0}(z)
$$

in the neighbourhood of $E_{\alpha 0}$, where $\widetilde{D}_{\alpha 0}(z)$ is less singular that $\left(E_{\alpha 0}-z\right)^{-1}$ It is easy to show that $N_{\alpha 0}$ must be a real number. Clearly (3.10) is equivalent to

$$
D_{\alpha 0^{-1}}=\frac{E_{\alpha 0}-z}{N_{\alpha 0}}+\bar{D}_{\alpha 0}(z)
$$

where $\overline{\bar{D}}_{\alpha 0}(z)$ goes to zero faster than $\left(E_{\alpha 0}-z\right)$ in the neighbourhood of $E_{\alpha \mathbf{0}}$.

Because $R(z)=(H-z)^{-1}$, it is obvious that $\langle\beta|R(z)| \alpha\rangle$ must have a pole on the real axis for the system to have a stationary state. Due to the form of (3.7) and the fact that $F_{\beta \alpha}(z)$ has at most a finite discontinuity, we can state the following:

Criterion I: It is only possible to construct a perturbed stationary state corresponding to the unperturbed state $|\alpha\rangle$ when $D_{\alpha}(z)$ has a pole on the real axis.

From our above assumptions this condition is fulfilled for $\lambda=0$. The corresponding perturbed stationary state will now be constructed. Let $|A\rangle$ be a arbitrary state in the Hilbert space defined by the complete set of unperturbed states, and define $\left|\psi_{\alpha}\right\rangle_{0}$ by

$$
\left\langle A \mid \psi_{\alpha}\right\rangle_{0}=N_{\alpha 0^{\frac{1}{2}}} \lim _{z \rightarrow E_{\alpha 0}^{-}}\left[D_{\alpha 0^{-1}}(z)\langle A|R(z)| \alpha\rangle_{0}\right],
$$

where the limit is taken through complex values of $z$. We prove that $\left|\psi_{\alpha}\right\rangle$ is a stationary state of the system for $\lambda=0$ with the energy $E_{\alpha \mathbf{0}}$. Since $\langle A|R(z)| \alpha\rangle_{0}$ is proportional to $D_{\alpha 0}(z)$ by (3.7), it is clear that the limit in (3.12) exists, and does not vanish. For simplicity we will assume that $E_{\alpha 0}$ does not lie on the cut of $F_{\beta \alpha 0}(z)$ so that this limit does not depend on the way in which the real axis is approached (otherwise the state obtained obeys 
the ingoing or outgoing wave condition depending on the side from which the real axis is approached $\left.{ }^{*}\right)$. The proof follows from the operator identity

$$
H R(z)=1+z R(z),
$$

whence

$$
\begin{aligned}
\left\langle A|H| \psi_{\alpha}\right\rangle_{0} & =N_{\alpha 0^{\frac{1}{3}}} \lim _{z \rightarrow E_{\alpha 0}{ }^{\prime}}\left[D_{\alpha 0} 0^{-1}(z)\langle A|H R(z)| \alpha\rangle_{0}\right] \\
& =E_{\alpha 0}\left\langle A \mid \psi_{\alpha}\right\rangle_{0} .
\end{aligned}
$$

It is easy to show that $\left|\psi_{\alpha}\right\rangle_{0}$ is normalized and reduces to $|\alpha\rangle$ in the limit of vanishing interaction.

It is now clear why Van Hove's method is so well suited to the investigation of the states of the system. As soon as the matrix elements of $R(z)$ are known in the unperturbed representation, it is straightforward to write explicit expressions for those stationary states which may be constructed by means of perturbation theory.

4. Criterion for metastable states. We are now in a position to state the conditions under which it is possible to construct a metastable perturbed state. We have already found a perturbed stationary state for $\lambda=0$, when $D_{\alpha 0}(z)$ lias a pole on the real axis. We shall now assume that $D_{\alpha}(z)$ has no pole for small $\lambda \neq 0$, but that it becomes very large on the real axis and reaches its maximum near $E_{\alpha 0}$ in a point of the real axis where $\operatorname{Im} D_{\alpha}(z) \neq 0$. In this case we can expect to find a metastable state for small $\lambda \neq 0$. We shall also assume that near $E_{\alpha 0}, D_{\alpha}(z)$ can be closely approximated by a function which can be analytically continued across the cut on the real axis, and when $D_{\alpha}(z)$ becomes large on the cut, that this behaviour manifests itself as a pole in the analytical continuation. When we treat the Lee model in section 7, we shall show explicitly how this pole in the analytical continuation arises.

We shall denote the analytical continuation of $D_{\alpha}(z)$ (in the approximate

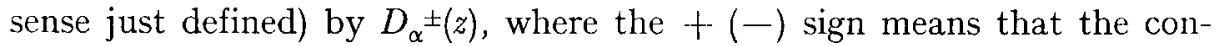
tinuation has been made from above (below) the cut on the real axis. Further we shall call the new sheets of the Riemann surface the $z^{ \pm \text {-sheets. }}$ By our assumptions we shall have a pole in each of these sheets, and shall further suppose that these poles occur at the points $z=E_{\alpha} \perp$ where

$$
E_{\alpha^{ \pm}}=\operatorname{Re} E_{\alpha} \mp i\left|\operatorname{Im} E_{\alpha}\right|,
$$

and $E_{\alpha}$ will be identified with the complex energy already considered in section 2 . We may now write

$$
D_{\alpha}^{ \pm}(z)=\frac{N_{\alpha^{ \pm}}}{E_{\alpha^{ \pm}}-z}+\tilde{D}_{\alpha^{ \pm}}(z),
$$

*) See reference 2 
where $\tilde{D}_{\alpha} \pm(z)$ is the rest of the Laurent expansion of $D_{\alpha}^{ \pm}(z)$ about $E_{\alpha} \pm$. Since $D_{\alpha}^{ \pm}(z)$ goes over into $D_{\alpha 0}(z)$ in the limit $\lambda \rightarrow 0$, we have

$$
\begin{aligned}
& \lim _{\lambda \rightarrow 0} N_{\alpha}^{ \pm}=N_{\alpha 0}, \\
& \lim _{\lambda \rightarrow 0} E_{\alpha^{ \pm}}=E_{\alpha 0} .
\end{aligned}
$$

We shall now distinguish two cases:

$$
\text { 1. } E_{\alpha 0}=a_{0} \quad \text { 2. } E_{\alpha 0}>a_{0} \text {, }
$$

where $a_{0}$ is the point at which the cut of $D_{\alpha}(z)$ begins for all $\lambda$. In case 1, $E_{\alpha 0}$ is a branch point for all $\lambda$, whereas in case $2, E_{\alpha 0}$ only becomes a branch point in the cut in the limit $\lambda \rightarrow 0$. In case 1 we must therefore have the additional requirement

$$
\operatorname{Re} E_{\alpha}>E_{\alpha 0},
$$

so that $D_{\alpha}(z)$ will become large on the cut. Clearly (4.5) is not necessary in case 2 . We shall also discuss the circles of convergence of (4.2), which we shall call $g_{\alpha}{ }^{ \pm}$and which both have the same radius $r_{\alpha}$. In case 1 , the branch point $E_{\alpha 0}$ is the nearest singularity to the poles at $E_{\alpha}{ }^{ \pm}$. Hence the circles $g_{\alpha}{ }^{ \pm}$are given by the equations

$$
\left|z-E_{\alpha}{ }^{ \pm}\right|=\left|E_{\alpha 0}-E_{\alpha}{ }^{ \pm}\right|=r_{\alpha} .
$$

The situation is pictured in figure 1. By (4.4)

$$
\lim _{\lambda \rightarrow 0} r_{\alpha}=0 .
$$

In case 2 , there is no such obvious limit to $r_{\alpha}$. However, we shall assume that (4.7) holds and we shall distinguish two subcases:

$$
\begin{array}{ll}
\text { 2a. } r_{\alpha}=\left|E_{\alpha 0}-E_{\alpha} \pm\right|, \quad & \lim _{\lambda \rightarrow 0} r_{\alpha}=0, \\
\text { 2b. } r_{\alpha}>\left|E_{\alpha 0}-E_{\alpha} \pm\right|, & \lim _{\lambda \rightarrow 0} r_{\alpha}=0 .
\end{array}
$$

In principle it is possible for $r_{\alpha}$ to be non-vanishing in the limit $\lambda \rightarrow 0$, but it does not restrict the generality to assume $r_{\alpha} \rightarrow 0$. In all cases, it is clear that the part of $g_{\alpha}{ }^{+}$above the real axis and the part of $g_{\alpha}{ }^{-}$below the real axis both lie in the original $z$-plane.

We also wish to discuss the requirement given by (2.4), which, with our identification of $E_{\alpha}$, becomes

$$
\left|\operatorname{Re} E_{\alpha} \pm\right| \gg\left|\operatorname{Im} E_{\alpha} \pm\right| .
$$

The physical basis of this condition follows from the uncertainty principle and the requirement that the uncertainty in the energy be much smaller than the energy itself. Since it is always possible to make a transformation which shifts the zero of energy, (4.8) can only be meaningful when $\operatorname{Re} E_{\alpha}{ }^{ \pm}$ is measured relative to a natural zero for the energy of the problem. In case 1 it is clear that the natural zero for the energy is $a_{0}$. In fact, when we treat 
the Fermi gas in section $8, a_{0}$ will turn out to be the ground state energy. Thus (4.8) implies that in this case we must make the new assumption

$$
\left|\operatorname{Re} E_{\alpha}-E_{\alpha 0}\right| \gg\left|\operatorname{Im} E_{\alpha}\right|,
$$

which, in terms of a limiting process, becomes

$$
\lim _{\lambda \rightarrow 0} \operatorname{Im} E_{\alpha} /\left(\operatorname{Re} E_{\alpha}-E_{\alpha 0}\right)=0 .
$$

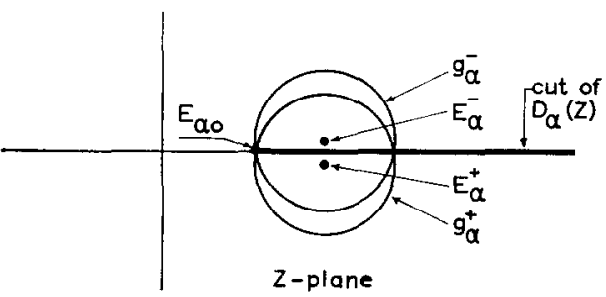

Fig. 1 . The circles $g_{\alpha} \pm$ for case 1 . In case 2 these circles need not pass through $E_{\alpha 0}$.

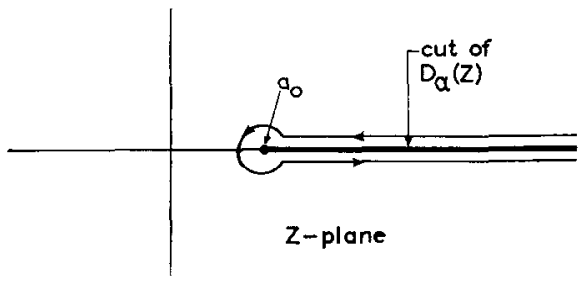

Fig. 2. The contour for evaluating $P_{\alpha}(t)$. The lines are along the upper and lower parts of the real axis.

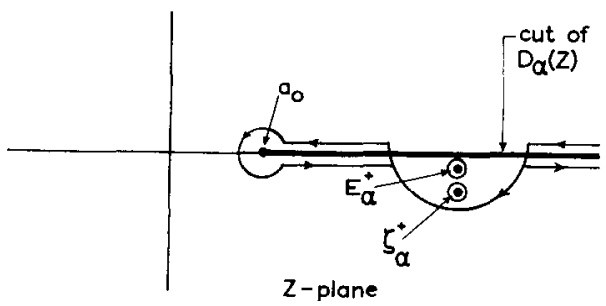

Fig. 3. The contour for $P_{\alpha}(t)$ after it has been deformed.

We shall see that (4.9) is automatically satisfied for the Fermi gas. In case 2 there is more ambiguity in the choice of a natural zero for the energy. In case 2a. we shall take it to be $E_{\alpha 0}$ and require that both (4.9) and (4.10) hold. In case $2 \mathrm{~b}$ we shall take it to be $a_{0}$, whence (4.8) follows from the fact that

$$
\lim _{\lambda \rightarrow 0} E_{\alpha}=E_{\alpha 0}>a_{0} .
$$

We may now summarize our assumptions in the following:

Criterion II: It is possible to construct a metastable perturbed state corresponding to the state $|\alpha\rangle$ when $D_{\alpha}(z)$ becomes very large near a point 
on its cut and can be approximated near this point by a function with analytical continuations across the cut having poles near the real axis.

That these conditions are sufficient for the construction of a metastable state will be demonstrated in the next three sections.

5. Ansatz for the metastable state. We shall now formulate the ansatz to be made for our metastable state $\left|\psi_{\alpha}\right\rangle$. To do this we introduce a complex number $\zeta_{\alpha}$, which is a function of $\lambda$ such that

$$
\begin{gathered}
\operatorname{Re} \zeta_{\alpha}=\operatorname{Re} E_{\alpha} \\
\left|\operatorname{Im} \zeta_{\alpha}\right| \gg\left|\operatorname{Im} E_{\alpha}\right| \\
\left|\operatorname{Im} \zeta_{\alpha}\right| \ll r_{\alpha} .
\end{gathered}
$$

Note that $\zeta_{\alpha}$ is by no means uniquely defined by these conditions. Further we introduce the complex numbers

$$
\zeta_{\alpha}^{ \pm}=\operatorname{Re} \zeta_{\alpha} \mp i\left|\operatorname{Im} \zeta_{\alpha}\right| \text {. }
$$

It follows from (5.1) and (5.3) that $\zeta_{\alpha}{ }^{ \pm}$lie within those portions of the circles $g_{\alpha} \mp$ which lie in the original $z$-plane (the upper (lower) signs are to be taken together). In terms of limiting processes these conditions become

$$
\begin{aligned}
& \lim _{\lambda \rightarrow 0} \operatorname{Im} E_{\alpha} / \operatorname{Im} \zeta_{\alpha}=0 \\
& \lim _{\lambda \rightarrow 0} \operatorname{Im} \zeta_{\alpha} / r_{\alpha}=0 .
\end{aligned}
$$

We also have

$$
\lim _{\lambda \rightarrow 0} \zeta_{\alpha}=E_{\alpha 0},
$$

which follows from (5.1), (5.3), and (4.7).

We shall take as our ansatz

$$
\left\langle A \mid \psi_{\alpha}\right\rangle \equiv N_{\alpha}^{\frac{1}{t}} D_{\alpha}^{-1}\left(\zeta_{\alpha}\right)\left\langle A\left|R\left(\zeta_{\alpha}\right)\right| \alpha\right\rangle,
$$

Where $N_{\alpha}$ is the normalization constant. Since $\zeta_{\alpha}$ is a complex number, no limit is needed in (5.8) as all of the functions involved are analitic away from the real axis (this is in contrast to (3.12)). By (5.7) and (3.12), we see that $\left|\psi_{\alpha}\right\rangle$ reduces to $\left|\psi_{\alpha}\right\rangle_{0}$ in the limit $\lambda \rightarrow 0$. To choose $N_{\alpha}$ we first compute $\left\langle\psi_{\alpha} \mid \psi_{\alpha}\right\rangle$. Using the partial fraction decomposition

$$
R\left(\zeta_{\alpha}{ }^{*}\right) R\left(\zeta_{\alpha}\right)=\left(\zeta_{\alpha}{ }^{*}-\zeta_{\alpha}\right)^{-1}\left[R\left(\zeta_{\alpha}{ }^{*}\right)-R\left(\zeta_{\alpha}\right)\right]
$$

and (3.7), we find easily that

$$
\left\langle\psi_{\alpha} \mid \psi_{\alpha}\right\rangle=N_{\alpha}\left(\zeta_{\alpha}{ }^{*}-\zeta_{\alpha}\right)^{-1}\left[D_{\alpha}^{-1}\left(\zeta_{\alpha}\right)-D_{\alpha}{ }^{-1}\left(\zeta_{\alpha}{ }^{*}\right)\right]\langle\alpha \mid \alpha\rangle .
$$

Since $\zeta_{\alpha}$ is a complex number, the coefficient of $\langle\alpha \mid \alpha\rangle$ is real and finite and we may choose

$$
N_{\alpha}=\left(\zeta_{\alpha}{ }^{*}-\zeta_{\alpha}\right)\left[D_{\alpha}{ }^{-1}\left(\zeta_{\alpha}\right)-D_{\alpha}{ }^{-1}\left(\zeta_{\alpha}{ }^{*}\right)\right]^{-1},
$$


whence $\left|\psi_{\alpha}\right\rangle$ is normalized, i.e.

It is easy to show that

$$
\left\langle\psi_{\alpha} \mid \psi_{\alpha}\right\rangle=\langle\alpha \mid \alpha\rangle
$$

$$
\lim _{\lambda \rightarrow 0} N_{\alpha}=N_{\alpha 0}
$$

6. Properties of $\varphi_{\alpha}(z)$. As the last step before calculating $P_{\alpha}(t)$, we shall discuss the function $\varphi_{\alpha}(z)$ defined by (3.3), taking for $\left|\psi_{\alpha}\right\rangle$ the ansatz (5.8). To compute it we use another partial fraction decomposition

$$
R\left(\zeta_{\alpha}{ }^{*}\right) R(z) R\left(\zeta_{\alpha}\right)=\frac{\left(\zeta_{\alpha}{ }^{*}-\zeta_{\alpha}\right) R(z)-\left(\zeta_{\alpha}-z\right) R\left(\zeta_{\alpha}\right)-\left(z-\zeta_{\alpha}\right) R\left(\zeta_{\alpha}{ }^{*}\right)}{\left(\zeta_{\alpha}{ }^{*}-\zeta_{\alpha}\right)\left(\zeta_{\alpha}{ }^{*}-z\right)\left(\zeta_{\alpha}-z\right)}
$$

together with (5.8), (3.7), (5.10) and (5.11), whence

$\varphi_{\alpha}(z)=\frac{\left(\zeta_{\alpha}{ }^{*}-\zeta_{\alpha}\right) D_{\alpha}(z)-\left(\zeta_{\alpha}{ }^{*}-z\right) D_{\alpha}\left(\zeta_{\alpha}\right)-\left(z-\zeta_{\alpha}\right) D_{\alpha}\left(\zeta_{\alpha}{ }^{*}\right)}{\left(\zeta_{\alpha}{ }^{*}-z\right)\left(\zeta_{\alpha}-z\right)\left[D_{\alpha}\left(\zeta_{\alpha}{ }^{*}\right)-D_{\alpha}\left(\zeta_{\alpha}\right)\right]}\left\langle\psi_{\alpha} \mid \psi_{\alpha}\right\rangle$.

This rather complicated form for $\varphi_{\alpha}(z)$ turns out to have simple properties. Its numerator vanishes for $z=\zeta_{\alpha}$ and $z=\zeta_{\alpha}{ }^{*}$. Thus $\varphi_{\alpha}(z)$ has no singularities at these points, and therefore has the same singularities as $D_{\alpha}(z)$. Thus with our ansatz for the state $\left|\psi_{\alpha}\right\rangle$, we need only know the properties of $D_{\alpha}(z)$ to compute $P_{\alpha}(t)$.

We shall now discuss the analytical continuations of $\varphi_{\alpha}(z)$, which we shall call $\varphi_{\alpha}^{ \pm}$. By (6.1), these continuations can be constructed once $D_{\alpha}^{ \pm}(z)$ are known, and the latter continuations were extensively discussed in section 4. We shall first choose $\zeta_{\alpha}=\zeta_{\alpha}{ }^{+}$so that $D_{\alpha}\left(\zeta_{\alpha}\right)=D_{\alpha}{ }^{-}\left(\zeta_{\alpha}{ }^{+}\right)$. With this choice, $\zeta_{\alpha}{ }^{*}==\zeta_{\alpha}{ }^{-}$and $D_{\alpha}\left(\zeta_{\alpha}\right)=D_{\alpha}{ }^{+}\left(\zeta_{\alpha}{ }^{-}\right)$. Thus using (6.1) we can write $\varphi_{\alpha}^{ \pm}(z)=\frac{\left(\zeta_{\alpha}{ }^{-}-\zeta_{\alpha}{ }^{+}\right) D_{\alpha}^{ \pm}(z)-\left(\zeta_{\alpha}-z\right) D_{\alpha}{ }^{-}\left(\zeta_{\alpha}{ }^{+}\right)-\left(z-\zeta_{\alpha}{ }^{+}\right) D_{\alpha}{ }^{+}\left(\zeta_{\alpha}{ }^{-}\right)}{\left(\zeta_{\alpha}{ }^{-}-z\right)\left(\zeta_{\alpha}{ }^{+}-z\right)\left[D_{\alpha}{ }^{+}\left(\zeta_{\alpha}{ }^{-}\right)-D_{\alpha}{ }^{-}\left(\zeta_{\alpha}{ }^{+}\right)\right]}\left\langle\psi_{\alpha} \mid \psi_{\alpha}\right\rangle$.

By (4.2), $\varphi_{\alpha}^{ \pm}(z)$ has poles at $z=E_{\alpha}^{ \pm}$in the $z^{ \pm-s h e e t s . ~ I t ~ a l s o ~ h a s ~ p o l e s ~ a t ~}$ $z=\zeta_{\alpha} \pm$ in the $z^{ \pm-s h e e t s, ~ s i n c e ~ t h e ~ n u m e r a t o r ~ o f ~}(6.1)$ does not vanish at these points. Thus the continuations of $\varphi_{\alpha}(z)$ do not have the same singularities as the continuations of $D_{\alpha}(z)$. Note that had we chosen $\zeta_{\alpha}=\zeta_{\alpha}{ }^{-}$, we would also have found the extra poles at $z=\zeta_{\alpha} \pm$. Therefore we can say that $\varphi_{\alpha}{ }^{ \pm}$has extra poles in the $z^{ \pm}$-sheets at the points $\zeta_{\alpha}^{ \pm}$, independently of the sign of $\operatorname{Im} \zeta_{\alpha}$. Finally we note that the part of $p_{\alpha}^{ \pm}(z)$ not proportional to $D_{\alpha} \pm(z)$ is the same in all sheets of the Riemann surface.

We can now use (4.2) to calculate $\varphi_{\alpha}^{ \pm}$within the circles $g_{\alpha}^{ \pm}$, After some tedious algebra, we find

$$
\begin{aligned}
& \varphi_{\alpha}^{ \pm}(z)=Q_{\alpha}^{ \pm}\left[\frac{1}{E_{\alpha}^{ \pm}-z}\left\{1-\frac{E_{\alpha}^{\mp}-E_{\alpha}^{ \pm}}{\zeta_{\alpha^{ \pm}}-E_{\alpha}^{ \pm}}\right\}+\frac{1}{\zeta_{\alpha}^{ \pm}-z}\left\{\frac{E_{\alpha}^{\mp}-E_{\alpha}^{ \pm}}{\zeta_{\alpha}^{\mp}-\zeta_{\alpha}^{ \pm}}+\frac{E_{\alpha}^{\mp}-\frac{E_{\alpha}^{ \pm}}{\zeta_{\alpha}^{ \pm}}-E_{\alpha}^{ \pm}}{\zeta^{\mp}}+\right.\right. \\
& \frac{\left(N_{\alpha}^{ \pm}-N_{\alpha}^{\mp}\right)\left(E_{\alpha}^{ \pm}-E_{\alpha}^{\mp}\right)}{N_{\alpha}^{ \pm}\left(\zeta_{\alpha}^{\mp}-\zeta_{\alpha}^{ \pm}\right)}+\frac{\left|E_{\alpha}^{\mp}-\zeta_{\alpha}^{ \pm}\right|^{2}}{N_{\alpha}^{ \pm}\left(\zeta_{\alpha}^{\mp}-\zeta_{\alpha}^{ \pm}\right)\left(\zeta_{\alpha}^{\mp}-z\right)}\left[\left(\zeta_{\alpha}^{\mp}-\zeta_{\alpha}^{ \pm}\right) \tilde{D}_{\alpha}^{ \pm}(z)-\right. \\
& \left.\left.\left.-\left(\zeta_{\alpha}^{\mp}-z\right) \tilde{D}_{\alpha}^{\mp}\left(\zeta_{\alpha}^{ \pm}\right)-\left(z-\zeta_{\alpha}^{ \pm}\right) \tilde{D}_{\alpha}^{ \pm}\left(\zeta_{\alpha}^{\mp}\right)\right]\right\}\right],
\end{aligned}
$$


where

$$
\begin{aligned}
&\left(Q_{\alpha}^{ \pm}\right)^{-1}=1+\frac{E_{\alpha}^{\mp}-E^{ \pm}}{\zeta_{\alpha}^{\mp}-\zeta_{\alpha}^{ \pm}}+\frac{\left(N_{\alpha}^{ \pm}-N_{\alpha}^{\mp}\right)\left(E_{\alpha}^{ \pm}-\zeta_{\alpha}^{\mp}\right)}{N_{\alpha}^{ \pm}\left(\zeta_{\alpha}^{\mp}-\zeta_{\alpha}^{ \pm}\right)}+ \begin{array}{c}
\left|E_{\alpha}^{\mp}-\zeta_{\alpha}^{ \pm}\right|^{2} \\
N_{\alpha}^{ \pm}\left(\zeta_{\alpha}^{\mp}-\zeta_{\alpha}^{ \pm}\right)
\end{array}\left[\tilde{D}_{\alpha}^{+}\left(\zeta_{\alpha}^{\mp}\right)\right. \\
&\left.\tilde{D}_{\alpha}^{\mp}\left(\zeta_{\alpha}{ }^{\mp}\right)\right] .
\end{aligned}
$$

7. Calculation of $P_{\alpha}(t)$. We have finally reached the point where we can use (3.2) to calculate $P_{\alpha}(t)$. Since the cut of $D_{\alpha}(z)$ begins at $a_{0}$, we shall evaluate $P_{\alpha}(t)$ around the contour given in figure 2 . We shall have to make the calculation separately for positive and negative times. For $t>0$, our program will be to deform the upper part of the contour into the $z^{+}$-sheet in the neighbourhood of $E_{\alpha 0}$, remaining inside $g_{\alpha}{ }^{+}$, whereas for $t<0$, we

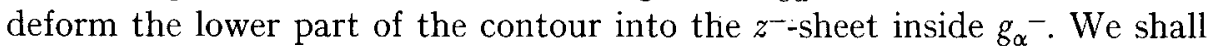
only do the calculation explicitly for $t>0$ as the calculation for $t<0$ follows from the symmetry of the analytical continuations about the real axis. We must again distinguish between the two cases defined in section 4 because the pole at $E_{\alpha}{ }^{+}$and the pole at $\zeta_{\alpha}+$ both go to $E_{\alpha 0}$ in the limit $\lambda \rightarrow 0$. In case 1 when $E_{\alpha 0}$ is a branch point, the contour can not necessarily be deformed inside $g_{\alpha}{ }^{+}$. It turns out that in this case the deformation is possible because of (4.9) and this is proved in the appendix. There is no difficulty in deforming the contour in case 2 . After the deformation has been shown to be possible, the calculation is identical for all cases and we shall give it explicitly only for case 2 .

We shall now deform the upper part of the contour in the neighbourhood of $E_{\alpha}$ into the $z^{+}$-sheet as is shown in figure 3. The semicircular part is chosen just within the circles $g_{\alpha}{ }^{+}$and $g_{\alpha}{ }^{-}$. For simplicity we shall also deform the lower part of the contour in the neighbourhood of $E_{\alpha 0}$ so that it lies in the original $z$-plane along the same semicircular path. The point of this deformation is that in the limit $\lambda \rightarrow 0$, the poles at $E_{\alpha}{ }^{+}$and $\zeta_{\alpha}{ }^{+}$will give a large contribution to the upper part of the contour; thus this contribution is evaluated by deforming the contour through the poles. We shall now write

$$
P_{\alpha}(t)=A_{\alpha}(t)+B_{\alpha}(t)+C_{\alpha}(t),
$$

where $A_{\alpha}(t)$ is the contribution from the two poles, $B_{\alpha}(t)$ comes from the sum of the contributions from the two semicircular parts, and $C_{\alpha}(t)$ is the contribution from the rest. With (6.3), $A_{\alpha}(t)$ can be integrated explicitly to give

$$
\begin{aligned}
& A_{\alpha}(t)=Q_{\alpha}+\left[\mathrm{e}^{-i t E_{\alpha}+}\left\{1-\frac{E_{\alpha}^{-}-E_{\alpha}^{+}}{\zeta_{\alpha}^{+}-E_{\alpha}{ }^{+}}\right\}+\mathrm{e}^{-i t \zeta_{\alpha}+}\left\{\frac{E_{\alpha}-E_{\alpha}^{+}}{\zeta_{\alpha}--\zeta_{\alpha}{ }^{+}}+\frac{E_{\alpha}-E_{\alpha}{ }^{+}}{\zeta_{\alpha}-E_{\alpha}{ }^{+}}+\right.\right. \\
& +\frac{\left(N_{\alpha}^{+}-N_{\alpha}{ }^{-}\right)\left(E_{\alpha}{ }^{+}-\zeta_{\alpha}^{-}\right)}{N_{\alpha}{ }^{+}\left(\zeta_{\alpha}{ }^{-}-\zeta_{\alpha}{ }^{+}\right)}+\frac{\mid E_{\alpha}{ }^{-}-\zeta_{\alpha}{ }^{2}}{N_{\alpha}{ }^{+}\left(\zeta_{\alpha}{ }^{-}-\zeta_{\alpha}{ }^{+}\right)} \text {. } \\
& \left.\left.\cdot\left[\tilde{D}_{\alpha}{ }^{+}\left(\zeta_{\alpha}{ }^{+}\right)-\tilde{D}_{\alpha}{ }^{-}\left(\zeta_{\alpha}{ }^{+}\right)\right]\right\}\right]\left\langle\psi_{\alpha} \mid \psi_{\alpha}\right\rangle \text {. }
\end{aligned}
$$


Since the parts of the contour contributing to $B_{\alpha}(t)$ and $C_{\alpha}(t)$ are the same in the two sheets but have opposite directions, only the part of $p_{\alpha}(z)$ that is proportional to $D_{\alpha}(z)$ can contribute (see (6.1) and (6.2)). Thus by also using (6.4)

$$
B_{\alpha}(t)=Q_{\alpha}{ }^{+} \frac{i\left|E_{\alpha}-\zeta_{\alpha}\right|^{2}}{2 \pi N_{\alpha}{ }^{+}} \int \mathrm{d} z \mathrm{e}^{-i t z} \frac{D_{\alpha}{ }^{+}(z)-D_{\alpha}-(z)}{\left(\zeta_{\alpha}^{-}-z\right)\left(\zeta_{\alpha}{ }^{+}-z\right)}\left\langle\psi_{\alpha} \mid \psi_{\alpha}\right\rangle,
$$

where the integral is taken in a clockwise direction on the semicircular path, and

$C_{\alpha}(t)=Q_{\alpha}{ }^{+} \frac{i\left|E_{\alpha}{ }^{-}-\zeta_{\alpha}{ }^{+}\right|^{2}}{2 \pi N_{\alpha}{ }^{+}} \int_{u_{0}}^{\infty} \mathrm{d} x \mathrm{e}^{-i t x} \frac{D_{\alpha}(x-i 0)-D_{\alpha}(x+i 0)}{\left|x-\zeta_{\alpha}{ }^{+}\right|^{2}}\left\langle\psi_{\alpha} \mid \psi_{\alpha}\right\rangle$,

where the integral is restricted so that the region of the $x$-axis is within the semicircular path is omitted. In (7.4) we have not included the contribution from the small circle centered on the branch point because it vanishes identically since we assume that $D_{\alpha}(z)$ is less singular than $\left(a_{0}-z\right)^{-1}$ in the neighbourhood of $a_{0}$. We also assume that the integral in (7.4) is convergent for large $x$.

It is now straightforward to establish (2.7) for $t>0$ by using the limiting process which we have denoted by LIM in section 2. With (5.5), (4.3), and the property that $D_{\alpha 0}(z)$ is less singular than $\left(E_{\alpha 0}-z\right)^{-1}$ for $z$ near $E_{\alpha 0}$, we find

and

$$
\lim _{\lambda \rightarrow 0} Q_{\alpha}^{ \pm}=1
$$

$$
\operatorname{LIM}\left[\exp \left(\text { it } \operatorname{Re} E_{\alpha}\right) A_{\alpha}(t)\right]=\exp \left(-t\left|\operatorname{Im} E_{\alpha}\right|\right)\left\langle\psi_{\alpha} \mid \psi_{\alpha}\right\rangle
$$

Along the semicircular path in the original $z$-plane, $D_{\alpha}(z)=D_{\alpha}^{-}(z)$. Thus it is straightforward to make the estimate

$$
\left|B_{\alpha}(t)\right| \leq M\left(\operatorname{Im} \zeta_{\alpha} / r_{\alpha}\right)
$$

where $M$ is finite in the limit. We can also write

$$
\left|C_{\alpha}(t)\right| \leq M\left(\operatorname{Im} \zeta_{\alpha}\right)^{2}
$$

since the integral in (7.4) is finite. Thus by using (5.6) and (5.7) we have

$$
\text { LIM }\left[\exp \left(i t \operatorname{Re} E_{\alpha}\right)\left\{B_{\alpha}(t)+C_{\alpha}(t)\right\}\right]=0 .
$$

It is obvious that these results will also hold for $t<0$ when the calculation is made by deforming into the $z^{-}$-sheet. This completes the proof of (2.7) under the conditions stated in section 4 and the ansatz (5.8).

In making this proof we have been very careful to point out that it is only necessary to make the analytical continuation in a very small neighbourhood around $E_{\alpha 0}$. This is a very important point because the function $D_{\alpha}(z)$ is determined completely by the discontinuity on its cut. Thus it would be 
possible to change this discontinuity slightly in such a way that the function itself is essentially unchanged, but that it is impossible to make an analytical continuation. Conversely since we need the analytical continuation only in a small region, it will be always possible to approximate $D_{\alpha}(z)$ in this region by a function whose analytical continuation exists. Thus the analytical continuation method is no more of a restriction than any other method which approximates $D_{\alpha}(z)$ in the region near $E_{\alpha 0}$.

In defining the limiting process LIM, we have only considered times of the order of $\left|\operatorname{Im} E_{\alpha}\right|^{-1}$, or, more precisely, times just large enough so that only the largest term in the series expansion of $\operatorname{Im} E_{\alpha}$ in terms of $\lambda$ is relevant in the limit. However, from the proof, it is immediately clear that our result holds for all times up to the order of $\left|\operatorname{Im} E_{\alpha}\right|^{-1}$, i.e. for $|t| \lesssim\left|\operatorname{Im} E_{\alpha}\right|^{-1}$. This rather remarkable fact can only be understood when it is emphasized that our result refers only to the limiting behaviour of the quantity $P_{\alpha}(t)$ for small $\lambda$, and that it is not necessary that the limiting value be approached uniformly in $t$. In fact it is easy to see that $C_{\alpha}(t)$ vanishes much more slowly than $B_{\alpha}(t)$. Thus if one were to construct a metastable state for a particular system by this method, the correction terms which would be present could (and probably would) be relatively more important for small times than for times of the order of $\left|\operatorname{Im} E_{\alpha}\right|^{-1}$. For times much larger than $\left|\operatorname{Im} E_{\alpha}\right|^{-1}$, it will be necessary to retain many terms of the expansion of $\operatorname{Im} E_{\alpha}$ in powers of $\lambda$, in which case the decay will not necessarily be exponential.

The final result which we have obtained shows that (2.7) holds independently of the sign of $\operatorname{Im} \zeta_{\alpha}$. Thus the question arises as to what extent the metastable state itself depends on the sign of $\operatorname{Im} \zeta_{\alpha}$. By continuity, the answer depends on whether the limiting state $\left|\psi_{\alpha}\right\rangle_{0}$ is a scattering state or not. If it is a scattering state, $\left|\psi_{\alpha}\right\rangle$ will obey the incoming or outgoing wave condition depending on whether $\operatorname{Im} \zeta_{\alpha}$ is negative or positive. If $\left|\psi_{\alpha}\right\rangle_{0}$ is a stationary state without scattering character, e.g. the ground state, then $\left|\psi_{\alpha}\right\rangle$ will depend on the sign of $\operatorname{Im} \zeta_{\alpha}$ only through terms very small for small $\operatorname{Im} \zeta_{\alpha}$.

8. The Lee model. We shall now apply our results to the Lee model to show that in this case our state reduces to the state defined by Glaser and Källén, and that our method yields a simple calculation of the decay spectrum. We shall not formulate the problem in detail as this has been done by Glaser and Källén. We shall use their notation. The calculation of the matrix elements of $R(z)$ is straightforward and yields

$$
\begin{aligned}
& \left\langle 1_{v}, 0,0|R(z)| 1_{v}, 0,0\right\rangle \equiv D_{v}(z)=\left[m_{v}-z-G_{v}(z)\right] 1 \\
& \left\langle 0,1_{N}, 1_{\bar{k}}|R(z)| 1_{v}, 0,0\right\rangle=\frac{g_{0}}{\sqrt{2 v \omega}} \frac{f(\omega)}{m_{N}+\omega-z} D_{v}(z)
\end{aligned}
$$




$$
\begin{aligned}
& \left\langle 0,1_{N}, 1_{\overline{k^{\prime}}}|R(z)| 0,1_{N}, 1_{k^{\prime}}^{-}\right\rangle=\frac{\delta_{\bar{k}^{\prime} k}}{m_{N}+\omega-z}+ \\
& \quad+\frac{g_{0}^{2}}{2 \eta} \frac{f\left(\omega\left(\bar{k}^{\prime}\right) f(\omega(\bar{k})) D_{v}(z)\right.}{\sqrt{\omega\left(\overline{k^{\prime}}\right) \omega(\bar{k})}} \frac{\left(m_{N}+\omega\left(\bar{k}^{\prime}\right)-z\right)\left(m_{N}+\omega(\bar{k})-z\right)}{},
\end{aligned}
$$

where in the limit of infinite volume

$$
G_{v}(z)=\frac{g_{0}^{2}}{4 \pi^{2}} \int_{\mu}^{\infty} \mathrm{d} \omega \frac{f^{2}(\omega) \sqrt{\omega^{2}-\mu^{2}}}{m_{N}+\omega-z} .
$$

We now assume that the cul-off function $f(\omega)$ is sufficiently well behaved so that it is possible to make analytical continuations of $G_{v}(z)$, which we shall call $G_{v}^{ \pm}(z)$. This can be achieved if necessary by approximating $f(\omega)$ in a proper neighbourhood by a more regular function. We seek a solution of the equation

$$
m_{v}-z-G_{v}^{\perp}(z)=0
$$

near the real axis. Following Glaser and Källén, we suppose that there is a number $M>m_{N}+\mu$ such that

$$
m_{v}-M-\operatorname{Re} G_{v}^{ \pm}(M)=0,
$$

and that the quantity

$$
\operatorname{Im} G_{v}^{ \pm}(M)= \pm \frac{g_{0}^{2}}{4 \pi} f^{2}\left(M-m_{N}\right) \sqrt{\left(M-m_{N}\right)^{2}-\mu^{2}}
$$

is small. Thus we can expand (8.5) about $z=M$, i.e.

$m_{v}-M-(z-M)-G_{v^{ \pm}}(M)-(z-M)\left\{\frac{\mathrm{d}}{\mathrm{d} z} G_{v^{ \pm}}(z)\right\}_{z=M}=0$.

so that $D_{v^{ \pm}}(z)$ has poles at

$E_{v^{ \pm}}=M \mp i \frac{g_{0}^{2}}{4 \pi} f^{2}\left(M-m_{N}\right) \sqrt{\left(M-m_{N}\right)^{2}-\mu^{2}}\left[1+\left\{\frac{\mathrm{d}}{\mathrm{d} z} G_{v^{ \pm}}(z)\right\}_{z=M}\right]^{-1}$.

It is easy to show that

$$
\left\{\frac{\mathrm{d}}{\mathrm{d} z} G_{v^{ \pm}}(z)\right\}_{z=M}
$$

are real numbers. With these assumptions, the Lee model becomes a particular example of case 2 as defined in section 4 .

Using (5.8) we can immediately write down the metastable state arising from the pole at $E_{v}{ }^{+}$. It is

$$
\begin{gathered}
\left|\psi_{v}\right\rangle=N_{v^{\mathrm{b}}} D_{v}^{-1}\left(\zeta_{v}\right)\left[\left|1_{v}, 0,0\right\rangle\left\langle 1_{v}, 0,0\left|R\left(\zeta_{v}\right)\right| 1_{v}, 0,0\right\rangle+\right. \\
\left.+\Sigma_{\bar{k}}\left|0,1_{N}, 1_{k^{-}}\right\rangle\left\langle 0,1_{N}, 1_{\bar{k}}\left|R\left(\zeta_{v}\right)\right| 1_{v}, 0,0\right\rangle\right]= \\
=N_{v^{\frac{1}{2}}}\left[\left|1_{v}, 0,0\right\rangle+\frac{g_{0}}{\sqrt{2 v}} \sum_{\bar{k}} \frac{f(\omega)}{\left(m_{N}+\omega-\zeta_{v}\right) \sqrt{ } \omega}\left|0,1_{N}, 1_{k}-\right\rangle\right],
\end{gathered}
$$


where according to (5.1) and (5.2) $\zeta_{v}$ is a complex number verifying.

$$
\operatorname{Re} \zeta_{v}=\operatorname{Re} E_{v}{ }^{+} \text {and }\left|\operatorname{Im} \zeta_{v}\right| \geqslant\left|\operatorname{Im} E_{v^{\dagger}}\right| \text {. }
$$

Here as in the general case the sign of $\operatorname{Im} \zeta_{v}$ is immaterial. By (5.3) we must also have

$$
\left|\operatorname{Im} \zeta_{v}\right| \ll r_{v} .
$$

It is easy to see that (8.10) is the same state that was obtained by Glaser and Källén. They also found the condition given by (8.11) but did not find (8.12). From (8.10) we see that $\operatorname{Im} \zeta_{v}$ is roughly the width of the unperturbed $\left|0,1_{N}, 1_{\bar{k}}\right\rangle$ states needed to expand $\left|\psi_{v}\right\rangle$. As will be presently shown $\operatorname{Im} E_{v}{ }^{+}$is the width of the decay spectrum fir the decay of $\left|\psi_{v}\right\rangle$ into the perturbed $\left|0,1_{N}, 1_{\vec{k}}\right\rangle$ states. Therefore the conditions given by (8.11) and (8.12) are physically quite reasonable.

To calculate the decay spectrum we need the perturbed stationary states. According to $\mathrm{Van} \mathrm{Hove}^{2}$ ), the states which obey the outgoing wave condition are given by

$$
\left\langle A \mid \psi_{\bar{k}}^{+}\right\rangle=\lim _{z \rightarrow m_{N}+\omega(\bar{k})+i 0}\left[\left(m_{N}+\omega(\bar{k})-z\right)\left\langle A|R(z)| 0,1_{N}, 1_{\bar{k}}\right\rangle\right] .
$$

We need to compute

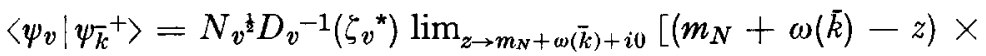

$$
\begin{aligned}
& \left.\times\left\langle 1_{v}, 0,0\left|R\left(\zeta_{v}{ }^{*}\right) R(z)\right| 0,1_{N}, 1_{\bar{k}}\right\rangle\right]= \\
& =-N_{v^{\frac{1}{2}}} \frac{g_{0} f(\omega)[2 v \omega]^{-\frac{1}{2}}}{\zeta_{v^{*}}-\left(m_{N}+\omega\right)} \frac{D_{v^{+}}\left(m_{N}+\omega\right)}{D_{v^{+}\left(\zeta_{v}^{*}\right)}{ }^{*}},
\end{aligned}
$$

which follows from (8.2) and the partial fraction decomposition that preceeds (5.9). In the limit of infinite volume the decay spectrum becomes

$$
\left|\left\langle\psi_{v} \mid \psi_{\bar{k}^{+}}{ }^{+}\right\rangle\right|^{2}=\frac{\left(\zeta_{v}{ }^{*}-\zeta_{v}\right) \operatorname{Im} G_{v}{ }^{+}\left(\omega+m_{N}\right)\left|D_{v}{ }^{+}\left(\omega+m_{N}\right)\right|^{2}}{\pi\left[D_{v}\left(\zeta_{v}{ }^{*}\right)-D_{v}\left(\zeta_{v}\right)\right] \mid \zeta_{v}{ }^{*}-\left(m_{N}+\omega\right)^{2}} \mathrm{~d} \omega
$$

with the use of (5.10) and (8.7). With (8.8) and (8.9) we find easily that when $\left(m_{N}+\omega\right)$ is close to $M$,

$$
\left|\left\langle\psi_{v} \mid \psi_{\bar{k}}^{+}\right\rangle\right|^{2} \simeq \frac{\left|\operatorname{Im} E_{v^{+}}\right| \mathrm{d} \omega}{\pi\left|m_{N}+\omega-E_{v^{+}}\right|^{2}},
$$

which is the expected shape of the decay spectrum, and shows that $\left|\operatorname{Im} E_{v}{ }^{+1}\right|$ is its width.

9. The Fermi gas. We shall now treat the Fermi gas as a model of a large system of nucleons principally to show how the parameter $\lambda$ has to be chosen. We shall not discuss this problem in detail as this has been done in a very clear fashion by Hugenholt $z$ *). We shall use his notation, and look at the

*) See reference 4, sections 2 and 14, and also references 13 and 14 . 
situation corresponding to the unperturbed state $|k ;\rangle$ with one nucleon of momentum $k$ in addition to the Fermi sea. From (3.8) we have

$$
D_{k}(z)=\left[\varepsilon_{0}+\varepsilon_{k}-z-G_{k}(z)\right]^{-1},
$$

where $\varepsilon_{0}$ is the unperturbed energy of the Fermi sea and $\varepsilon_{k}$ is the unperturbed energy of the extra particle. For real $x$, it is possible to write

$$
\lim _{\eta \rightarrow 0} G_{k}(x \pm i|\eta|)=K_{k}(x) \pm i J_{k}(x) .
$$

Hugenholtz has shown that $J_{k}(x)$ is nonvanishing only for $x \geq E_{0}+E_{F}$, and that for $|k|=k_{F}$ and $0<x-E_{0}-E_{F} \ll E_{F}$

$$
J_{k}(x) \propto\left(x-E_{0}-E_{F}\right)^{2},
$$

where $k_{F}$ is the Fermi momentum, $E_{0}$ the perturbed energy of the Fermi sea, and $E_{F}$ the perturbed Fermi energy. He has also shown that $D_{k}(z)$ has a pole at $z=E_{0}+E_{F}$ when $|k|=k_{F}$, i.e.

$$
\varepsilon_{0}+\varepsilon_{k_{F}}-E_{0}-E_{F}-K_{k_{F}}\left(E_{0}+E_{F}\right)=0 .
$$

Thus this situation is a particular example of case 1 as defined in section 4 .

Again we assume that it is possible to define $G_{k}^{ \pm}(z)$ and look for a solution of the equation

$$
\varepsilon_{0}+\varepsilon_{k}-z-G_{k^{ \pm}}(z)=0
$$

near the real axis. Letting $z=x \mp i y$, we expand (9.4) about $z=x$ and obtain

$$
\varepsilon_{0}+\varepsilon_{k}-x \pm i y-G_{k^{ \pm}}(x) \pm i y\left\{\frac{\mathrm{d}}{\mathrm{d} z} G_{k^{ \pm}}(z)\right\}_{z=x}=0
$$

by neglecting higher powers of $y$. The imaginary part of (9.6) yields

$$
y=\left[1+\left\{\frac{\mathrm{d}}{\mathrm{d} z} G_{k}^{ \pm}(z)\right\}_{z=x}\right]^{-1} J_{k}(x),
$$

which according to (9.3) will be small when $x-E_{0}-E_{F} \ll E_{F}$. To solve the real part of (9.6), we choose a point on the Fermi surface which has the same angular coordinates as $k$. Then we can write

$$
K_{k}(x)=K_{k_{F}}(x)+\left(\varepsilon_{k}-\varepsilon_{k_{F}}\right)\left[\frac{\partial}{\partial \varepsilon_{k}} K_{k}(x)\right]_{k=k_{F}}+\ldots
$$

Using (9.4), the real part of (9.6) becomes

$$
\left(\varepsilon_{k}-\varepsilon_{k_{F}}\right)-\left(x-E_{0}-E_{F}\right)-\left(\varepsilon_{k}-\varepsilon_{k_{F}}\right)\left[\frac{\partial}{\partial \varepsilon_{k}} K_{k}(x)\right]_{k=k_{F}}+\ldots=0 .
$$

To first approximation

$$
x-E_{0}-E_{F} \propto\left(\varepsilon_{k}-\varepsilon_{k_{k}}\right) \equiv \lambda_{k},
$$


and $\lambda_{k}$ is clearly the small parameter in the problem. From (9.7) and (9.3) we see that

$$
y \propto \lambda_{k}^{2} .
$$

Thus $D_{K^{+}}(z)$ will have a pole in the $z^{+}$-sheet at $E_{k}{ }^{+}$, where

$$
\operatorname{Re} E_{k}{ }^{+}-E_{F}-E_{0} \propto \lambda_{k} \text { and } \operatorname{Im} E_{k}{ }^{+} \propto \lambda_{k}^{2},
$$

when $\lambda_{k}$ is small.

According to our general results, it is therefore possible to construct a metastable state corresponding to the unperturbed state $|k ;\rangle$ when $\lambda_{k}$ is small, i.e. when $k$ is close to the Fermi surface. Using the diagram methods developed by Hugenholt $z$ and (5.8), it is possible to write out an expression for this state in terms of diagrams. We will not do this explicitly. Finally we wish to note that the requirement given by (4.9) is automatically satisfied in this case.

11. Acknowledgements. The author is pleased to express his indebtedness to Professor L. Van Hove, who suggested this problem and whose searching criticism was most helpful at all stages of the work and in the preparation of the manuscript. He also wishes to thank Professor Van Hove and the rest of the staff of the Instituut voor Theoretische Fysica, particularly Miss E. Wesenhagen, for their especially kind hospitality. Finally he wishes to thank Professor N. G. van Kampen for many stimulating discussions.

$\mathrm{He}$ is indebted to the National Science Foundation for the award of a post-doctoral fellowship for the academic year 1958-1959, and to the 'Stichting voor Fundamenteel Onderzoek der Materie', which is supported by the Netherlands Organization for Pure Scientific Research (Z.W.O.) for financial support during the academic year 1959-1960. Finally he wishes to thank his wife for her constant encouragement.

\section{APPENDIX}

To show that we can deform the contour near $E_{\alpha 0}$ in case 1, we introduce the new complex variable $z$ by the transformation $*$ )

where

$$
z=E_{\alpha 0}+H_{\alpha}(1+\check{z})
$$

$$
H_{\alpha}=\operatorname{Re} E_{\alpha}-E_{\alpha 0}>0,
$$

the inequality following from (4.5). By (4.9) we have

$$
\lim _{\lambda \rightarrow 0} \operatorname{Im} E_{\alpha} / H_{\alpha}=0 .
$$

*) We are indebted to Professor Van Kampen for suggesting this transformation. 
In terms of the new variable, the branch point occurs at $z=-1$, and the poles in the $z^{+}$-sheet at $E_{\alpha}{ }^{+}$and $\zeta_{\alpha}{ }^{+}$occur respectively at $z_{1}=-i \operatorname{Im} E_{\alpha} / H_{\alpha}$ and $\ddot{z}_{2}=-i \operatorname{Im} \zeta_{\alpha} / H_{\alpha}$. By (3) and (5.6), both poles are close to the origin for amall $\lambda \neq 0$. Since the branch point is at $\check{z}=-1$, it is therefore possible to deform the contour into the $z^{+}$-sheet for small $\lambda+0$. After the transformation (1) has been made, one finds the quantity $H_{\alpha} z t$ in the exponential in the integrand of $P_{\alpha}(t)$ where previously one had $z t$. Because of (3) and (4.4), $\left|H_{\alpha} t\right| \rightarrow \infty$ in the limiting process when $|t| \rightarrow \infty$, and $\left|H_{\alpha} t\right| \rightarrow 0$ in the limiting process when $|t|$ remains finite. Thus it is clear that the proofs given in section 7 go through in case 1 just as for case 2 .

Received 30-6-60

REFERENCES

1) Van Hove, L., Physica ஹ1 (1955) 901.

2) Van Hove, L., Physica 22 (1956) 343.

3) Frazer, W. R. and Van Hove, L., Physica 24 (1958) 137.

4) Hugenholtz, N. M., Physica 23 (1957) 481.

5) Glazer, V. and Källén, G, Nuclear Phys. a (1957) 706.

6) Zumino, B., N.Y.U. Research Report No. CX 23. (1956).

7) Hohler, G., Zeitschr. f. Phys. 152 (1958) 546.

8) Okabayashi, T. and Sato, S., Progr. theor. Phys. 17 (1957) 30.

3) Araki, H., Munakata, Y., Kawaguchi, M., and Gotô, T., Progr. theor. Phys, 17 (1957) 419.

10) Naito, K., Progr. theor. Phys. 18 (1957) 200 and 18 (1957) 614.

11) Nakanishi, N., Progr. theor. Phys. 19 (1958) 607 and $\mathbf{2 0}$ (1958) 822.

12) Van Hove, L., Physica $\mathbf{2 3}$ (1957) 441.

13) Hugenholtz, N. M., Physica 23 (1957) 533.

14) Hugenholtz, N. M. and Van Hove, L., Physica $\mathbf{9 4}$ (1958) 363. 